\title{
FLORÍSTICA EM ÄREAS DA MARGEM DIREITA DO BAIXO JACUÍ, RS, BRASIL ${ }^{1}$
}

\author{
Olinda Leites Bueno 1 \\ Márcia Therezinha Menna Barreto das Neves ${ }^{2}$ \\ Maria de Lourdes Abruzzi Aragão de Oliveira ${ }^{1}$ \\ Rosa Lúcia Dutra Ramos ${ }^{2}$ \\ Teresia Strehl ${ }^{1}$
}

Recebido em 20.10.86. Aceito em 06.02.87

RESUMO - O trabalho descreve a vegetação florestal e campestre situada à margem direita do baixo Jacuí, no Estado do Rio Grande do Sul. É apresentada uma lista com 441 táxons pertencentes a 84 famílias botânicas, constatados na área de estudo.

Palavras-chave: Composição florística; Floresta Estacional Decidual Aluvial; Vegetação de Margem.

\begin{abstract}
Floristic at the right margin of the Lower Jacui river, RS, Brazil.) This paper describes the forestal and fieldy vegetation at the right margin of the lower Jacui river, in the State of Rio Grande do Sul. A list with 441 taxa that belong to 84 botanic families observed in the studied area is presented.

Key words: Floristic composition; Seasonal Alluvial Deciduous Forest; Margin vegetation.
\end{abstract}

\section{Introdução}

Durante o ano de 1982, realizou-se um levantamento florístico na área prevista para a instalação do Pólo Carboquímico do Rio Grande do Sul, situada à margem direita do baixo rio Jacuí, próximo à confluência com o rio Taquari, na região fisiográfica da Depressão Central do Estado.

Os pontos selecionados para a amostragem da vegetação localizam-se entre as coordenadas geográficas $29^{\circ} 57^{\prime} 23^{\prime \prime}$ - latitude Sul e $51^{\circ} 46^{\prime} 24^{\prime \prime}$ longitude Oeste (Fig. I). Geomorfologicamente, os terrenos localizados nesta área correspondem a planícies aluviais e a coxilhas pampeanas (INCRA, 1972 e 1973).

As planícies aluviais apresentam altitudes entre menos $10 \mathrm{~m}$ e $30 \mathrm{~m}$, ocorrem ao longo do rio Jacuí, do arroio do Conde e outros cursos d'água da região.

\footnotetext{
1'Técnico Superior Pesquisador da Fundação Zoobotânica do Rio Grande do Sul e Bolsista do $\mathrm{CNPq}$ - Av. Salvador França, 1427, Caixa Postal 1188. CEP 90610, Porto Alegre, RS.

2 Técnico Superior Pesquisador da Fundação Zoobotânica do Rio Grande do Sul.
} 
Os solos predominantes são do tipo "planossol", Vacacaí (BRASIL X RIO GRANDE DO SUL, 1970).

As coxilhas pampeanas atingem $80 \mathrm{~m}$ de altitude, sendo os solos predominantes do tipo "laterítico humo avermelhado distrófico", nome regional São Jerônimo (BRASIL X RIO GRANDE DO SUL, 1970).

O clima corresponde a variedade "Cfalg", do tipo subtropical (MOTTA, 1951 e KOEPPEN, 1948) com chuvas bem distribuídas.

LINDMAN (1906) refere-se às matas dolongodo rio Jacuí desde as corredeiras de Cachoeira até a foz do rio Guaíba chamando-as de "matas de anteparo ou de mata marginal".

A vegetação da região foi pouco estudada até o momento. SAINT-HILAIRE (1939) menciona a passagem pelo Jacuí, quando de sua viagem ao Rio Grande do Sul. RAMBO (1956), assim descreve a fisionomia da região: "na região carbonífera, entre Butiá e Arroio dos Ratos, as coxilhas, engrossando pouco a pouco em direção à raiz da Serra, se acham revestidas de um tapete gramináceo muito uniforme, repartido por fracas formações de galeria e porções insignificantes de mata brejosa. Nas partes mais altas, a serra do terreno supera a da Campanha, nutrindo uma flora muito baixa e pobre, de legítimos xerófitos. $\mathrm{Na}$ beira do Arroio dos Ratos, a mata de anteparo se alarga, vindo terminar nas formações palustres que caracterizam o curso inferior do Jacuí".

Estudos florísticos e ecológicos junto à margem esquerda do rio Jacuí foram realizados, em 1978, pelos pesquisadores da Fundação Zoobotânica do Rio Grande do Sul, na área de influência do III Pólo Petroquímico (Fundação Zoobotânica do Rio Grande do Sul, 1981).

\section{Material e métodos}

Para a amostragem da flora foram selecionadas áreas de campo e de mata, constituindo-se estas últimas em matas galeria e capões, localizadas nos municípios de São Jerônimo e Butiá (fig. 1).

Para o estudo dos campos estabeleceram-se três estratos: estrato superior de $0,80 \mathrm{~m}$ até $2 \mathrm{~m}$ de altura; estrato médio de $0,40 \mathrm{~m}$ até $0,70 \mathrm{~m}$ de altura; estrato inferior até $0,30 \mathrm{~m}$ de altura.

Para o estudo das florestas estabeleceram-se quatro estratos: estrato arbóreo, constituído pelas espécies emergentes e incluindo as espécies que formam o dossel; estrato das arvoretas; estrato arbustivo e estrato herbáceo.

As plantas coletadas foram determinadas, com auxílio de literatura especializada e confirmadas com consulta a especialistas após, foram integradas ao acervo do Herbário Prof. Dr. Alarich R.H. Schultz (HAS) do Museu de Ciências Naturais da Fundação Zoobotânica do Rio Grande do Sul.

\section{Resultados}

Embora não se tenha um cálculo planimétrico da área total ocupada pelos campos, matas e capōes, pode-se dizer, seguramente, que, nessa região, pelo menos $70 \%$ da área está representada pelos campos e 30\% pelas florestas. 

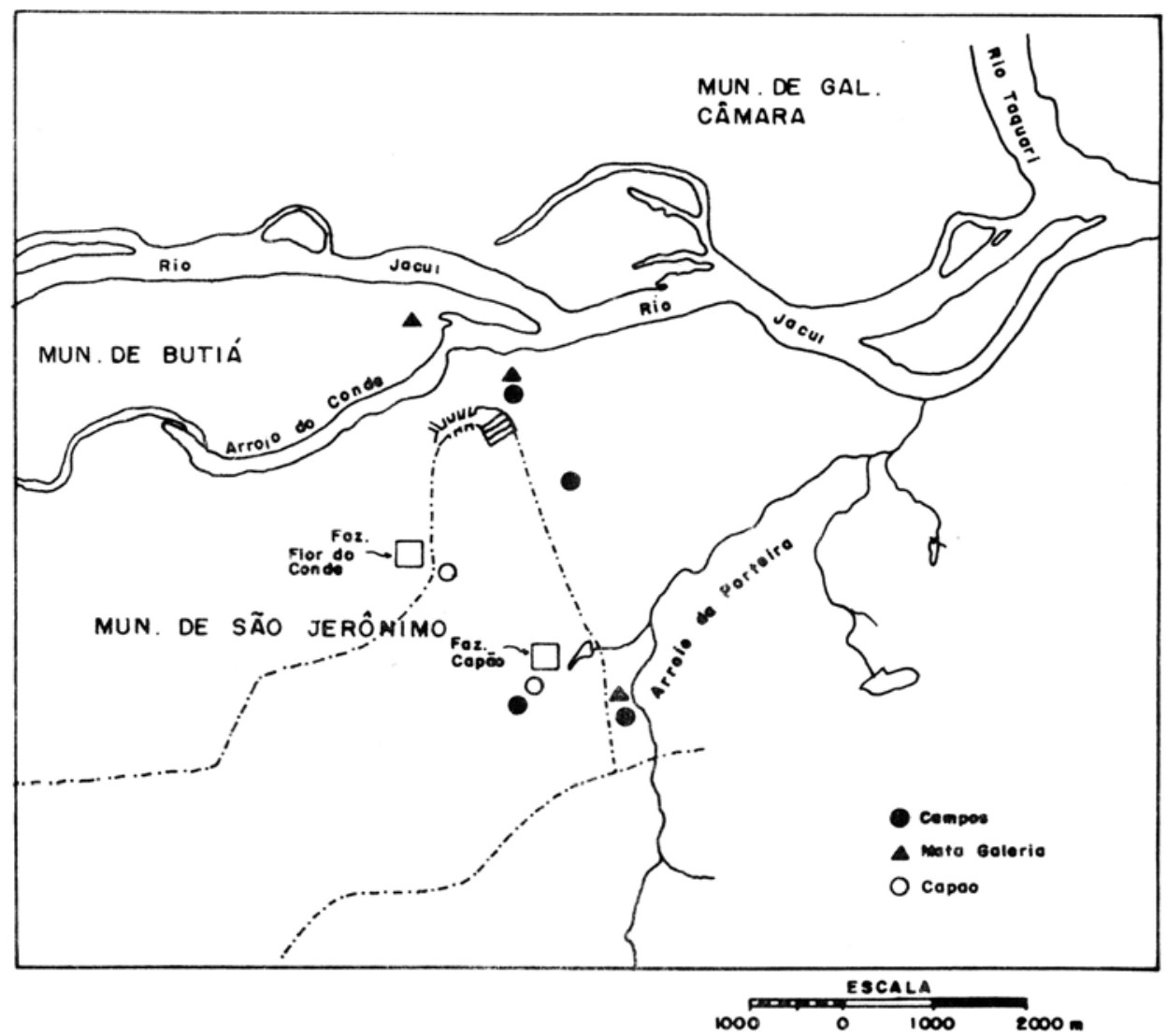

FIGURA 1 - Mapa da área de estudo com os pontos de coleta.

Em diferentes condições topográficas e ecológicas - brejos, várzeas aluviais e encostas das elevações - distinguem-se diferentes agrupamentos de espécies, sejam elas características do solo freável das encostas ou dos solos desde úmidos até alagados das baixadas. O aspecto fisionômico dessas áreas varia, também, com a estação do ano e o uso anterior e atual das mesmas.

Os campos das várzeas, anteriormente utilizados para cultivo do arroz, estão agora cobertos principalmente por Gramíneas, Ciperáceas, Onagráceas e Juncáceas entre outras famílias. Dentre as Gramíneas, nota-se o predomínio de Ischaemum minus e Paspalum pumilum, o primeiro com uma cobertura entre $80-90 \%$. 
Nos campos situados nas encostas e submetidos, até recentemente, a culturas cíclicas, constata-se a presença de espécies pioneiras muito agressivas, podendo destacar-se: Axonopus fissifolius, Cynodon dactylon, Eragrostis neesii, Paspalum notatum, Piptochaetium montevidense, Sida rhombifolia e Vernonia nudiflora.

Em áreas já há mais tempo sem cultivo, verifica-se a ocorrência de Andropogon lateralis, Aristida circinalis, A. filifolia, Eryngium horridum e Setaria geniculata.

De uma maneira geral, as encostas estão cobertas em $70 \%$ a $90 \%$ por Paspalum notatum; além desse encontram-se também no estrato baixo Aspilia montevidensis, Axonopus fissifolius, Desmodium incanum, Piptochaetium montevidense, Richardia brasiliensis e $R$. grandiflora. No estrato médio predominam Andropogon lateralis, Aristida laevis, A. jubata e Baccharis trimera. Pelo seu porte, formando o estrato alto, sobressaem Eryngium horridum e Senecio brasiliensis. Em solos arenosos, formando manchas esparsas, observa-se Peltodon longipes $\mathrm{e}$, com menor freqüência, Paspalum plicatulum.

Nos topos das colinas ocorrem com maior abundância espécies herbáceas ou arbustivas altas como Andropogon lateralis, Eryngium horridum, Sida rhombifolia e Vernonia nudiflora. Chama a atenção pela coloração da inflorescência e pelo porte, Eryngium ciliatum. Formando o estrato médio destacam-se Aristida jubata e A. laevis; no tapete graminoso baixo predomina Paspalum notatum, observando-se manchas de Piptochaetium montevidense acompanhado, com menor freqüência, por Axonopus fissifolius, Cynodon dactylon e Eragrostis neesii.

Nas áreas brejosas e várzeas aluviais, como componentes do estrato baixo, ocorrem Axonopus fissifolius, Ischaemum minus, Paspalum pumilum, $P$. notatum e Setaria geniculata. Caracterizando o estrato médio destacando-se Andropogon lateralis, Hypogynium virgatum e Rottboellia selloana. Eryngium pandanifolium constitui o estrato alto, também, fazendo parte desse, em alguns locais, Panicum grumosum.

Em locais mais encharcados, com água aflorando à superfície, encontram-se Eleocharis spp., Luziola peruviana, Rhynchospora emaciata, $R$. tenuis, Scleria hirtella, Utricularia tricolor, $U$. cf. erectiflora e $U$. cf. obtusa, além de Cyperus lanceolatus e Juncus spp.

Matas galeria e capōes

A vegetação florestal ainda existente na área limita-se a matas galeria e capões. Ass margens do rio Jacuí a vegetação é densa, atingindo cerca de $15 \mathrm{~m}$ de altura, havendo, em alguns locais, nítida predominância de Inga uruguensis.

O estrato superior é consituído principalmente por Luehea divaricata, Ruprechtia laxiflora, Vitex magapotamica e Matayba elaeagnoides. Embora não muito freqüentes destaca-se a presença de Ficus organensis e $F$. luschnathiana.

As espécies mais freqüentes, no estrato das arvoretas, são Sebastiania klotzschiana, Myrciaria tenella, Pouteria salicifolia, Guettarda uruguensis, Campomanesia rhombea e C. xanthocarpa. 
No estrato arbustivo predominam Daphnopsis racemosa, Pavonia sepium, Maytenus dasyclados, $M$. ilicifolia e Psychotria carthagenensis.

O estrato herbáceo é bastante descontínuo. Predominam Diodia brasiliensis, Oplismenus setarius, Ichnanthus pallens e, nos lugares mais úmidos, ocorrem Rhynchospora hieronymi, Anemia phyllitidis e Commelina cf. erecta.

Os epífitos são abundantes: várias Orquidáceas como Campylocentrum aromaticum, Oncidium fimbriatum, $O$. uniflorum e uma espécie de Eurystyles, várias Polipodiáceas e dentre as Bromeliáceas o gênero Tillandsia é o melhor representado, com várias espécies.

Dentre as lianas foram constatadas como mais freqüentes as seguintes: Calea pinnatifida, Doxantha unguis-cati, Pithecoctenium echinatum, Paullinia elegans, Combretum fruticosum, Smilax campestris e várias espécies de Mikania e Passiflora.

Às margens dos cursos d'água de menor porte, como o arroio da Porteira, a vegetação é mais baixa, atingindo o estrato superior a altura de $10 \mathrm{~m}$. A constituição em espécies é basicamente a mesma da mata galeria do rio Jacuí, podendo-se destacar como espécies emergentes Syagrus romanzoffiana e agrupamentos de Bambusa trinii. Como representantes da Floresta Atlântica (KLEIN, 1983) ocorrem Myrcia glabra no estrato superior e Faramea marginata e Bactris lindmaniana no estrato arbustivo.

Junto ao arroio do Conde, próximo a desembocadura no rio Jacuí, há uma diminuição da densidade das espécies arbóreas, assumindo a vegetação o aspecto de parque, destacando-se Pouteria gardnerana, Inga uruguensis e Nectandra megapotamica. Salix humboldtiana ocorre raramente. O solo, arenoso, está na maior parte descoberto, sendo o estrato inferior constituído por gramíneas, uma espécie de Zephyranthes (Amaryllidaceae) que ocorre com abundância $\mathrm{e}$, junto às margens d'água, destaca-se Mollugo verticillata (Aizoaceae).

Em áreas a maior parte do ano inundadas, observam-se agrupamentos de Cephalantus glabratus, Sebastiania schottiana e Phyllanthus sellowianus.

Em encostas e depressões do terreno, junto a sedes de fazendas, numa, paisagem onde predominam os campos, encontram-se resquícios de matas, formando capões. Nesses, o estrato superior que atinge até $16 \mathrm{~m}$ de altura é dominado por Patagonula americana e Cordia ecalyculata. Também são freqüentes nesse estrato, Parapiptadenia rigida, Luehea divaricata, Pouteria gardnerana e Cabralea cangerana. Duas espécies representantes da Floresta Atlântica (KLEIN, 1983) - Ficus organensis e Guapira opposita foram observadas.

No estrato das arvoretas destacam-se Trichilia clausseni, Guarea macrophylla, Casearia silvestris, Rollinia silvativa e Scutia buxifolia.

O estrato arbustivo é bastante alterado, sendo as espécies mais freqüentes Psychotria leiocarpa e Daphnopsis racemosa.

No estrato herbáceo ocorrem Pseudoechinolaena polystachya, Petiveria alliacea, Chaptalia nutans, Hydrocotyle leucocephala; nos bordos Sida carpinifolia e Elephantopus mollis, entre outros.

Os epífitos também são abundantes nos capões, destacando-se várias espécies de Tillandsia recurvata, Microgramma squamulosa e $M$. vacciniifolia, Peperomia blanda e $P$. catharinae e Rhipsalis baccifera. 
Dentre as liana Seguieria guaranitica é bastante comum no interior dos capões e Paullinia elegans ocorre preferencialmente nas margens.

No presente levantamento constatou-se a ocorrência de 441 táxons específicos e infraespecíficos, distribuídos em 84 famílias e 264 gêneros. Nas matas, foram mais representativas as famílias Myrtaceae, Leguminosae e Rubiaceae. Já nos campos, predominaram as famílias Compositae, Gramineae, Leguminosae e Cyperaceae.

As espécies são listadas na Tabela 1, em ordem alfabética de família, assinalando-se com um $\mathrm{X}$ sua ocorrência em campos, matas galeria ou capões.

TABELA I - Ocorrência das espécies (x) - campos e florestas

Florestas

Nome científico

Ncme popular

Campos

mata
galeria capão

ACANTHACEAE

Dicliptera sp.

Hygrophila brasiliensis (Spreng.) Lindau

Justicia axillaris (Nees.) Lindau

Ruellia sanguinea Griseb.

$\mathrm{x}$

$\mathrm{X}$

$\mathrm{x}$

AIZOACEAE

Mollugo verticillata $\mathrm{L}$.

$\mathrm{X}$

\section{ALISMATACEAE}

Echinodorus grandiflorus (Cham. et

chapéu-de-couro

$\mathbf{X}$

Schlecht.) Mich.

Sagittaria montevidensis Cham. et

Schlecht.

AMARANTHACEAE

Alternanthera philoxeroides (Mart.) Griseb.

Alternanthera tenella Colla

Iresine diffusa H. \& B. ex Willd.

Pfaffia tuberosa (Spreng.) Hicken

$\mathbf{x}$

$\mathrm{x}$

$\mathrm{x}$

AMARYLLIDACEAE

Zephyranthes sp.

X

ANNONACEAE

Rollinia silvatica (St. Hil.) Mart.

araticum, quaresma

$\mathrm{X}$

APOCYNACEAE

Macrosiphonia petraea (St. Hil.) K. Schum.

ARISTOLOCHIACEAE

Aristolochia sessilifolia (Klotzsch)

Malme

jarrinha-rasteira

$\mathbf{X}$ 
Florestas

Nome científico

Nome popular

Campos

$\underset{\text { mata }}{\text { galeria capão }}$

\section{BIGNONIACEAE}

Amphilophium vauthieri DC.

Clytostoma callistegioides (Cham.)

Bur. \& K. Schum.

Doxantha unguis-cati (L.) Miers

Jacaranda micrantha Cham.

Macfadyena dentata $\mathrm{K}$. Schum.

Pithecoctenium echinatum (Jacq.) Baill.

Tecoma stans (L.) Kunth

cipó-d'alho

cipo-unha-de-gato

caroba

pente-de-macaco

caroba-amarela

louro-salgueiro

guajuvira $\mathrm{x} \quad \mathrm{x}$

$\mathrm{x}$

X

$\mathrm{x}$

$\mathrm{x}$

$\mathrm{X}$

\section{$\mathbf{x}$}

$\mathrm{x}$

X

X

ananás (silvestre)

gravatá-zebra

cravo-do-mato

cravo-do-mato

cravo-do-mato

barba-de-pau

BROMELIACEAE

Smith

Ananas bracteatus (Lindl.) Schultes

Bilbergia zebrina (Herb.) Lindl.

Tillandsia aeranthos (Loisel.) L.B.

Smith

Tillandsia geminiflora Brong.

Tillandsia recurvata (L.) L.

Tillandsia stricta Soland.

Tillandsia usneoides (L.) L.

\section{CACTACEAE}

Rhipsalis bacciffera (Miller) Stearn.

\section{CAMPANULACEAE}

Pratia hederacea (Cham.) G. Don

Triodanis biflora (R. et Pav.) Grene

Wahlenbergia linarioides (Lam.) A.DC.

CARYOPHYLLACEAE

Arenaria cf. lanuginosa (Michx.) Rohrb.

Cerastium dicrotrichum Fenzl. ex Rohrb.

Drymaria cordata (L.) Willd.

Paronychia chilensis DC.

\section{CELASTRACEAE}

Maytenus dasyclados Mart.

Maytenus ilicifolia Mart. ex Reiss.

cancorosa;

sombra-de-touro

\section{COMBRETACEAE}

Combretum fruticosum (Loefl.) Stuntz. Terminalia australis Camb. 


\section{Florestas}

Nome científico

Nome popular

Campos

mata

galeria capão

\section{COMMELINACEAE}

Commelina cf. erecta L.

Commelina virginica $\mathrm{L}$.

Commelina sp.

Floscopa glabrata (Kunth) Hassk.

Tradescantia cf. fluminensis Vell.

trapoeiraba

$\mathrm{x}$

carrapicho-rasteiro $\mathrm{x}$

macela $\mathrm{x}$

mal-me-quer $\quad \mathbf{x}$

(silvestre)

Aster squamatus (Spreng.) Hieron.

Baccharidastrum triplinervium (Less.)

Cabr.

Baccharis anomala DC.

Baccharis crispa Spreng.

Baccharis dracunculifolia DC.

Baccharis illinita DC.

Baccharis microcephala (Less.) DC.

Baccharis pseudotenuifolia T'eodoro

Baccharis riograndensis Teodoro \& Vidal

Baccharis spicata (Lam.) Baill.

Baccharis trimera (Less.) DC.

Calea pinnatifida Less.

Chaptalia nutans (L.) Polak.

Chaptalia runcinata H.B.K.

Cirsium vulgare (Savi) Ten

Conyza blakei (Cabr.) Cabr.

Conyza chilensis Spreng.

Conyza cf. floribunda H.B.K.

Eclipta megapotamica (Spreng.) Sch.

Bip. ex Blake

Elephantopus mollis H.B.K.

Eupatorium arnottianum Griseb.

Eupatorium commersonii (Cass.) Hieron.

Eupatorium inulaefolium H.B.K.

Eupatorium laevigatum Lam.

Eupatorium umbelliforme Dusén. ex Malme

Facelis retusa (Lam.) Sch. Bip.

Gamochaeta cf. falcata (Lam.) Cabr.

Gochnatia polymorpha var. ceanothifolia $\mathbf{x}$

$x$

$x$ vassoura-comum $\quad \mathrm{x}$

$\mathrm{x}$

$\mathrm{x}$

$\mathrm{x}$

$\mathrm{x}$

$\mathrm{x}$

carqueja $\quad$ x

língua-de-vaca

$\mathbf{x}$

língua-de-vaca $\quad x$

$\mathbf{x}$

$\mathrm{x}$

$\mathrm{x}$

$\mathbf{x}$

$\mathbf{x}$

suçuaiá

$\mathrm{x}$

x

$x$

$x$

$\mathrm{x}$

$x$

$\mathrm{x}$

$x$

$\mathrm{x}$

x

$\mathbf{x}$

Hypochoeris brasiliensis (Less.) Benth.

Hypochoeris megapotamica Cabr.

$\mathrm{x}$

Hypochoeris sp.

Jaegeria hirta (Lag.) Less.

Jungia sellowii Less.

Lucilia nitens Less. 


\begin{tabular}{|c|c|c|c|c|}
\hline \multirow[b]{2}{*}{ Nome científico } & \multirow[b]{2}{*}{ Nome popular } & \multirow[b]{2}{*}{ Campos } & \multicolumn{2}{|c|}{ Florestas } \\
\hline & & & $\begin{array}{c}\text { mata } \\
\text { galeria }\end{array}$ & capão \\
\hline Mikania cf. cynanchifolia $\mathrm{H}$. et A. & & $\mathrm{x}$ & & \\
\hline Mikania cf. dusenii Robin. & & & $\mathrm{x}$ & \\
\hline Mikania cf. involucrata $\mathrm{H}$. et A. & cipó-sem-nome & & $\mathrm{x}$ & \\
\hline Mikania sp. & & $\mathrm{x}$ & & \\
\hline Noticastrum calvatum (Baker) Cuatr. & & $\mathrm{x}$ & & \\
\hline Noticastrum cf. sericeum (Less.) Phil. & & $\mathrm{x}$ & & \\
\hline Pterocaulon angustifolium DC. & & $x$ & & \\
\hline Pterocaulon polypterum (DC.) Cabr. & & $\mathrm{x}$ & & \\
\hline Pterocaulon polystachyum DC. & & $\mathrm{x}$ & & \\
\hline Pterocaulon virgatum (L.) DC. & & $\mathrm{x}$ & & \\
\hline Senecio bonariensis $\mathrm{H}$. et $\mathrm{A}$. & & $\mathrm{x}$ & & \\
\hline Senecio brasiliensis (Spreng.) Less. & maria-mole & $\mathrm{x}$ & & \\
\hline Senecio crassiflorus (Poir.) DC. & & $\mathrm{x}$ & & \\
\hline Senecio leptolobus DC. & & $\mathrm{x}$ & & \\
\hline Senecio cf. oligophyllus Baker & & $\mathrm{x}$ & & \\
\hline Senecio selloi (Spreng.) DC. & & $\mathrm{x}$ & & \\
\hline Solidago chilensis Meyen & & $\mathrm{x}$ & & \\
\hline Soliva pterosperma (Juss.) Less. & roseta & $\mathrm{x}$ & & \\
\hline Spilanthes arnicoides DC. & & $\mathrm{x}$ & & \\
\hline Stenachaenium campestre Baker & & $\mathrm{x}$ & & \\
\hline Stenachaenium macrocephalum (DC.) & & $\mathrm{x}$ & & \\
\hline Benth. et Hook. & & & & \\
\hline Trixis stricta (Spreng.) Less. & & $\mathrm{x}$ & & \\
\hline Vernonia brevifolia Less. & & $\mathrm{x}$ & & \\
\hline Vernonia flexuosa Sims & & $\mathrm{x}$ & & \\
\hline Vernonia megapotamica Spreng. & & $\mathrm{x}$ & & \\
\hline Vernonia nudiflora Less. & alecrim-do-campo & $\mathrm{x}$ & & \\
\hline Vernonia platensis (Spreng.) Less. & & $\mathrm{x}$ & & \\
\hline Vernonia sellowii Less. & & $\mathrm{x}$ & & \\
\hline Vernonia tweediana Baker & mata-pasto & & $\mathrm{x}$ & $x$ \\
\hline CONVOLVULACEAE & & & & \\
\hline Aniseia argentina (N.E.Brown) O’Donell & & $\mathrm{x}$ & $\mathrm{x}$ & \\
\hline Evolvulus sericeus Sw. & & $\mathrm{x}$ & & \\
\hline Ipomoea grandifolia (Dammer) O'Donell & & $\mathrm{x}$ & & \\
\hline Ipomoea sp. & & $\mathrm{x}$ & $\mathrm{x}$ & \\
\hline Merremia dissecta (Persoon) Hallier & & & $\mathrm{x}$ & \\
\hline CRUCIFERAE & & & & \\
\hline Lepidium aletes Macbr. & & $\mathrm{x}$ & & \\
\hline CYPERACEAE & & & & \\
\hline Bulbostylis capillaris (L.) Kunth & & $\mathrm{x}$ & & \\
\hline Cyperus corymbosus Rottb. & & $\mathrm{x}$ & & \\
\hline Cyperus entrerianus (Boeckl.) Barros & & $\mathrm{x}$ & & \\
\hline Cyperus haspan L. & & $\mathrm{x}$ & & \\
\hline Cyperus incomptus Kunth & & & $x$ & \\
\hline Cyperus lanceolatus Poir. & & $x$ & & \\
\hline Cyperus polystachyos Rottb. & & $\mathrm{x}$ & & \\
\hline Cyperus reflexus Vahl & & $\mathrm{x}$ & & \\
\hline
\end{tabular}


Florestas

Nome científico

Nome popular

Campos

$\underset{\text { galeria }}{\text { mata }}$

Cyperus rotundus $\mathrm{L}$.

junça $\quad x$

Cyperus sesquiflorus (Torr.) Mattf. \&

Kükenth.

Eleocharis cf. bonariensis Nees.

Eleocharis elata Boeckl.

Eleocharis nodulosa (Roth) Schult.

Eleocharis cf. viridans Kükenth.

Rhynchospora cf. corymbosa (L.) Britt.

Rhynchospora emaciata (Nees.) Boeckl.

Rhynchospora cf. glauca Vahl

Rhynchospora hieronymi Boeckl.

Rhynchospora cf. rostrata Lindm.

Rhynchospora tenuis Link

Scleria hirtella $\mathrm{Sw}$.

$\mathrm{x}$
$\mathrm{x}$
$\mathrm{x}$

$x$

tiririca $\mathrm{x}$

$\mathrm{x}$

$\mathbf{X}$

$\mathrm{x}$

$\mathrm{x}$

$\mathrm{x}$

\section{EUPHORBIACEAE}

Acalypha multicaulis Muell. Arg.

Actinostemon concolor (Spreng.) Muell. laranjeira-do-mato

$\mathbf{x}$

$\mathrm{x}$

Arg.

Croton dusenii Croizat

Euphorbia papillosa St. Hil.

Euphorbia selloi (Kl. et Gke.) Boiss.

Phyllanthus sellowianus Muell. Arg.

Sapium glandulatum (Vell.) Pax

Sebastiania klotzschiana (Muell. Arg.)

Muell. Arg.

Sebastiania schottiana (Muell. Arg.)

Muell. Arg.

$x$

leiteira $\quad \begin{aligned} & \mathbf{x} \\ & \mathbf{x}\end{aligned}$

sarandi-vermelho $\quad \mathrm{x}$

$\begin{array}{lll}\text { branquilho } & \mathrm{x} & \mathrm{x} \\ \mathrm{x} & \mathrm{x}\end{array}$

amarilho-branco; $\quad \mathrm{x}$

branquilho

Sebastiania serrata Muell. Arg.

\section{FLACOURTIACEAE}

Casearia silvestris $\mathrm{Sw}$.

chá-de-bugre

GRAMINEAE

Agrostis montevidensis Spreng. ex Nees. Andropogon bicornis $\mathrm{L}$.

Andropogon lateralis Nees.

Andropogon selloanus (Hack.) Hack.

Andropogon ternatus (Spreng.) Nees.

Aristida circinalis Lindm.

Aristida filifolia (Arech.) Herter

Aristida jubata (Arech.) Herter

Aristida laevis (Nees.) Kunth

Avena barbata Pott ex Link

Axonopus affinis Chase

Axonopus argentinus Parodi

Axonopus compressus ( $\mathrm{Sw}$.) $\mathrm{Pal}$. de

Beauv.

capim-mimoso $\quad \mathrm{x}$

macega $x$

capim-caninha $\mathrm{x}$

plumas-brancas $\quad \mathrm{x}$

barba-de-bode $x$

barba-de-bode $x$

capim-barba-de-bode $\quad \mathrm{x}$

barba-de-bode $x$

aveia-barbada $\mathrm{x}$

grama-tapete $\mathrm{x}$

grama-tapete $\mathrm{x}$

Axonopus fissifolius (Raddi) Kuhlm.

Axonopus sp.

(de folha larga)

grama-fina 
Nome científico

Nome popular

Florestas

Nome científico

Campos $\frac{\text { Florestas }}{\substack{\text { mata } \\ \text { galeria }}}$

$\bar{B}$ ambusa trinii Nees.

Briza calotheca (Trin.) Hack.

Briza subaristata Lam.

Briza subaristata var. subaristata Lam.

Cynodon dactylon (L.) Persoon

Danthonia sp.

Echinochloa crusgalli (L.) Pal. de

Beauv.

Eleusine tristachya (Lam.) Lam.

Eragrostis airoides Nees.

Eragrostis bahiensis (Schrad. ex

Schult.) Schult.

Eragrostis hypnoides (Lam.) Britt.

Eragrostis lugens Nees.

Eragrostis lugens Nees. var. glabrata

Nome popular

$\mathrm{x}$

taquaraçu

capim-trigo

$\mathrm{x}$

$\mathrm{x}$

$x$
$x$

grama-paulista $\quad \mathrm{x}$

capim-arroz $\quad \mathrm{x}$

pé-de-galinha $\quad x$

capim-açu $\quad \mathrm{x}$

Doell

Eragrostis neesii Trin. var. neesii

Eragrostis neesii Trin. var. lindmanii

(Hack.) Ekman.

Eragrostis plana Nees.

Erianthus cf. trinii (Hack.) Hack.

Eriochloa punctata (L.) Desv.

Hypogynium virgatum (Desv.) Dandy

Ichnanthus pallens (Sw.) Munro ex

Benth.

Ischaemum minus Presl

Luziola peruviana Gmel.

Olyra micrantha H.B.K.

Oplismenus setarius (Lam.) Roem. \&

Schultes

Panicum aquaticum Poir.

Panicum grumosum Nees.

Panicum helobium Mez ex Ekman.

Panicum laxum Sw.

Panicum milioides Nees.

Panicum ovuliferum Trin.

Panicum prionitis Nees.

Panicum stoloniferum Poir.

Panicum trichanthum Nees.

Panicum sp.

Paspalum arenarium Schrad.

Paspalum conjugatum Berg.

Paspalum notatum Fluegge

Paspalum pauciciliatum (Parodi) Herter

Paspalum paucifolium J. Swallen

Paspalum plicatulum Michx.

Paspalum pumilum Nees.

capim-mimoso-do-

banhado

taquarinha; cresciúme

grama-de-ponta

capim-canivão

capim-mimoso-do-

banhado

capim-santa-fé

capim-gordo;

capim-azedo

grama-forquilha

capim-sanduva

coqueirinho

palha-branca $\mathrm{x}$

$\mathrm{x}$
$\mathrm{x}$

$x$

$\mathrm{X}$

X

$\mathrm{x}$

$\mathrm{x}$

$\mathrm{x}$

X 


\section{Florestas}

Nome científico

Nome popular

Campos $\begin{gathered}\text { mata } \\ \text { galeria capão }\end{gathered}$

Paspalum urvillei Steud.

Paspalum vaginatum $\mathrm{Sw}$.

Phalaris sp.

Piptochaetium montevidense (Spreng.)

Parodi

Pseudoechinolaena polystachya (H.B.K.)

$x$.

grama-rasteira

$x$

capim-cabelo-de-porco $\quad x$

O. Stapf

Rhynchelytrum repens (Willd.) Hubbard

Rhynchoryza subulata (Ness.) Baill.

Rottboellia selloana Hack.

Schizachyrium condensatum (H.B.K.)

Nees.

Schizachyrium microstachyum (Desv.)

Ros.

Setaria geniculata (Lam.) Beauv.

Stipa neesiana Trin. et Rupr.

HYPOCRATEACEAE

Pristimeria andina Miers

\section{HYPOXIDACEAE}

Hypoxis decumbens $\mathrm{L}$.

capim-gafanhoto $\quad \mathrm{x}$

capim-rabo-de- $\quad x$

lagarto

capim-rabo-de-burro $\quad \mathrm{x}$

capim-rabo-de-raposa $\quad \mathrm{x}$

flechilha-violácea $\quad \mathrm{x}$

\section{ICACINACEAE}

Citronela paniculata (Mart.) Howard."

\section{IRIDACEAE}

Cypella sp.

Sisyrinchium sp.

JUNCACEAE

Juncus cf. capillaceus Lam.

Juncus cf. venturianus Castillón

cabelo-de-porco $\quad \mathrm{x}$

\section{LABIATAE}

Hyptis brevipes Poiteau

Hyptis fasciculata ssp.

fastigiata (Benth.) Harley

Hyptis cf. floribunda Briq. ex Micheli

Hyptis lorentziana O. Hoffm.

Peltodon longipes St. Hil.

Scutellaria platensis Spegaz.

Scutellaria racemosa Persoon

$\mathrm{x}$
$\mathrm{x}$

\section{LAURACEAE}

Nectandra megapotamica (Spreng.) $\mathrm{Mez}$

Nectandra rigida (H.B.K.) Nees.

canela-preta

canela-amarela

hortelā-do-mato

$\mathbf{X}$

canela-guaicá

canela-lageana

$\begin{array}{ll}\mathrm{x} & \mathrm{x} \\ \mathrm{x} \\ \mathrm{x}\end{array}$




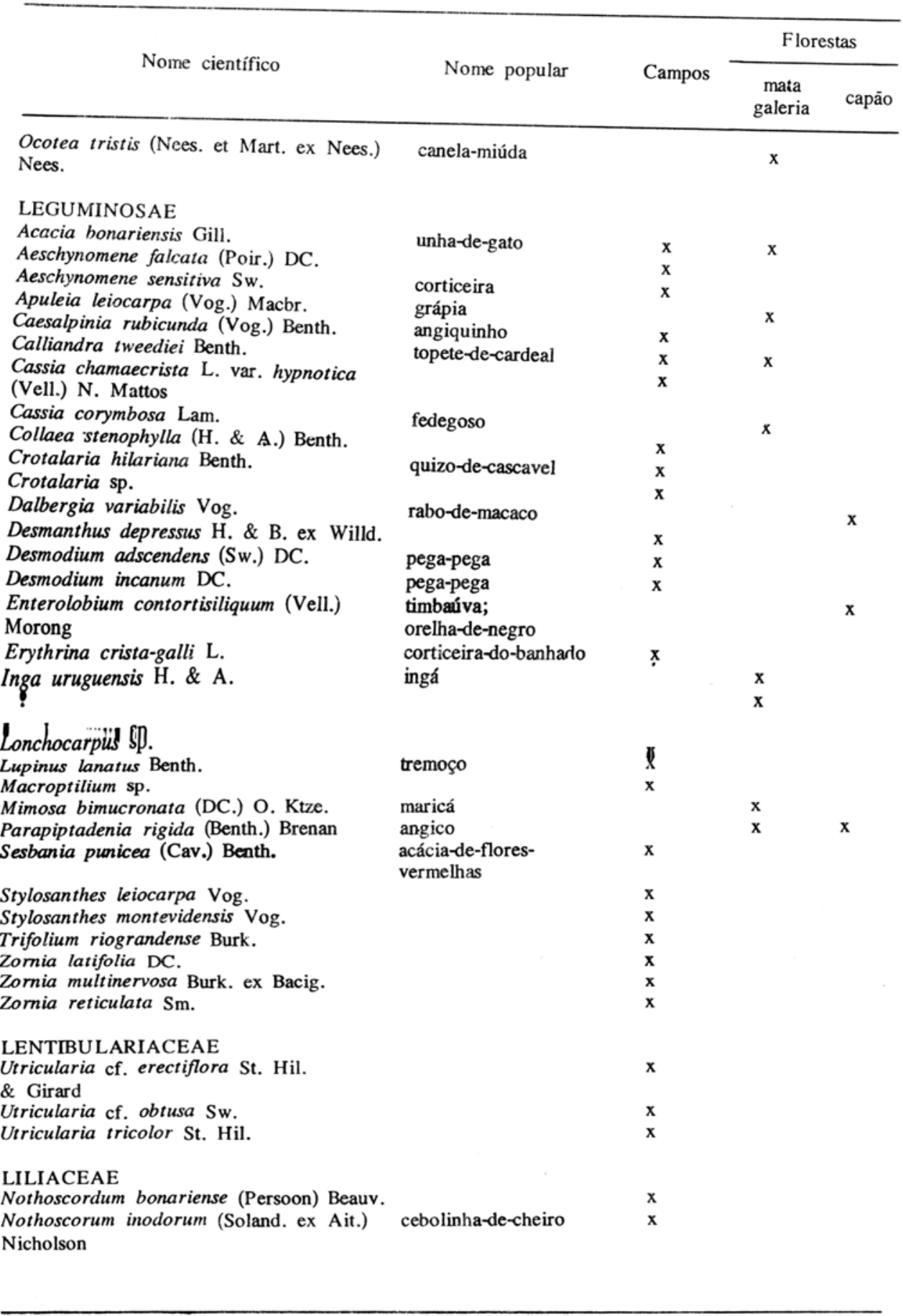




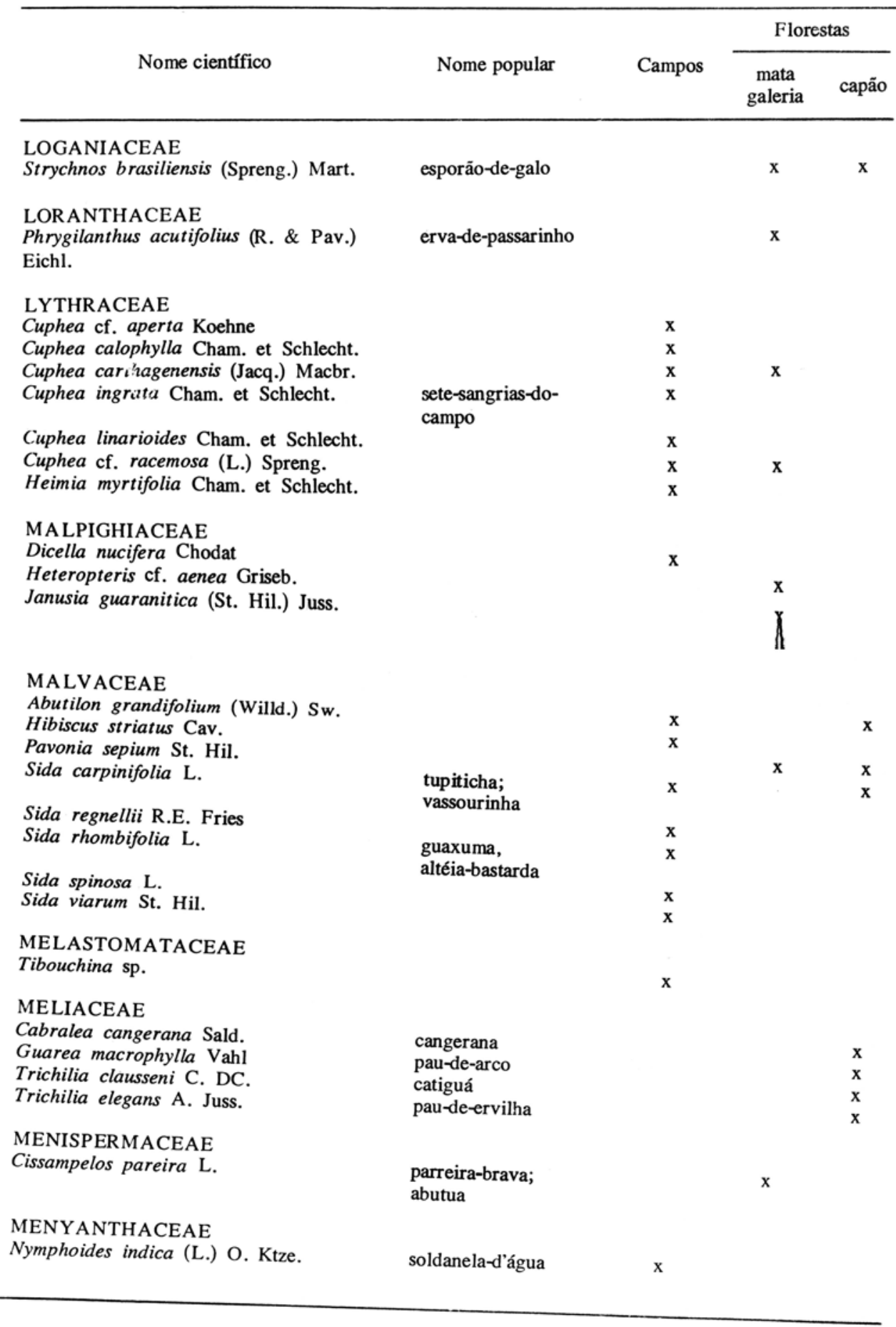


Florestas

Nome científico

Nome popular

Campos

mata
galeria capão

\section{MORACEAE}

Coussapoa schottii Miq.

Ficus luschnathiana (Miq.) Miq.

Ficus organensis (Miq.) Miq.

Sorocea bonplandii (Baill.) Burger,

Lanjow \& Boer

figueira; mata-pau

figueira-de-folha-

miúda

cincho; canapicica-de-

$\mathrm{x}$

folha-miúda

\section{MYRSINACEAE}

Rapanea lorentziana $\mathrm{Mez}$

capororoca;

Rapanea umbellata (Mart. ex A.DC.)

capororocão

capororoca;

peroba-d'água

\section{MYRTACEAE}

Blepharocalyx apiculatus Berg

Blepharocalyx salicifolius (H.B.K.) Berg

cambuim

Blepharocalyx tweediei (H. et A.) Berg

pitangueira-do-

$\mathbf{X}$

banhado

Calyptranthes concinna DC.

Campomanesia rhombea Berg

Campomanesia xanthocarpa Berg

pintangueira-do-mato

guabirobeira-de-

X

folha-miúda

guabirobeira-de-

folha-grande

Eugenia emarginata Glaziou

$x$

$x$

Eugenia hiemalis Camb.

Eugenia mansoni Berg

Eugenia pyriformis Camb.

Eugenia schüchiana Berg

Eugenia speciosa Camb.

Eugenia uniflora L.

Eugenia uruguayensis Camb. var.

uruguayensis

miúda

Eugenia uruguayensis Camb. var. opaca (Berg) Legr.

Eugenia vincaefolia Berg

Gomidesia palustris (DC.) Legr.

Myrceugenia glaucescens (Camb.) Legr.

et Kausel

Myrcia glabra (Berg) Legr.

Myrcia multiflora (Lam.) DC.

Myrciaria cuspidata Berg

Myrciaria tenella (DC.) Berg

ovaia; uvaia; uvalha

$\mathrm{x}$

pitanga

batinga-vermelha

uvá-de-facho

ubá, uvá

cambuim; cambuí

camboimzinho;

cambuí 
Florestas

Nome cientifico

Nome popular

Campos mata capão

\section{ONAGRACEAE}

Ludwigia sp.

$\mathbf{x}$

$\mathbf{x}$

ORCHIDACEAE

Campylocentrum aromaticum B. Rodr.

Eurystyles sp.

Galeandra beyrichii Rchb. f.

Habenaria sp.

Oncidium frimbriatum Lindl.

Oncidium uniflorum Booth.

\section{OXA LIDACEAE}

Oxalis bipartita St. Hil. ssp bipartita

Oxalis conorrhiza (Feuillé) Jacquin

Oxalis cf. papilionacea Hoffmgg.

Oxalis triangularis St. Hil. ssp.

triangularis

azedinha

tucum

x

$\mathbf{x}$

coqueiro

\section{PASSIFLORACEAE}

Passiflora cf. leptoclada Harms

Passiflora misera H.B.K.

Passiflora tenuifila Killip

maracujá

maracujá

$\mathbf{x}$

$\mathrm{x}$

$\mathrm{x}$

X

guiné; erva-pipi

cipó-limoeiro-

do-mato

$\mathbf{x}$

$\mathbf{x}$

tanchagem-miúda

$\mathbf{x}$

$\mathrm{x}$

$\mathbf{x}$

Polygala brasiliensis L.
Polygala leptocaulis Torr. \& Gray

Polygala brasiliensis L.
Polygala leptocaulis Torr. \& Gray

PIPERACEAE

Peperomia blanda (Jacq.) H.B.K.

Peperomia catharinae Miq.

PLANTAGINACEAE

Plantago myosurus Lam.

POLYGALACEAE

$x$

$x$ 
Florestas

Nome científico

Nome popular

Campos

mata

galeria capão

\section{POLYGONACEAE}

Coccoloba sp.

Polygonum punctatum Ell.

Ruprechtia laxiflora Meissn.

erva-de-bicho

$x$

viraru

$\mathrm{x}$

$\mathrm{x}$

POLYPODIACEAE

Microgramma squamulosa (Kaulf.) de la

cipó-cabeludo

$\mathrm{X}$

Sota

Microgramma vacciniifolia (Langsd. \& cipó-cabeludo

$\begin{array}{cc}\mathrm{x} & \mathrm{x} \\ \mathrm{x} & \mathrm{x} \\ \mathrm{x} & \mathrm{x} \\ \mathrm{x} & \mathrm{x} \\ \mathrm{x} & \end{array}$

Polypodium angiustum (H.B.W.) Liebm.

Polypodium squalidum Vell.

Polypodium cf. hirsutissimum Raddi

\section{PONTEDERIACEAE}

Eichhornia azurea (Sw.) Kunth

Pontederia lanceolata Nutt.

aguapé

$\mathrm{x}$

aguapé

$\mathrm{x}$

\section{PORTULACACEAE}

Talinum paniculatum (Jacq.) Gaertn.

beldroega-grande

\section{PTERIDACEAE}

Doryopteris multipartita (Fée) Sehnem

\section{RHAMNACEAE}

Gouania ulmifolia H. \& A.

Scutia buxifolia Reissek

coronilha

RANUNCULACEAE

Ranunculus flagelliformis Smith

rainúnculo-brasileiro

\section{RUBIACEAE}

Borreria eryngioides Cham. et Schlecht. Borreria fastigiata (Griseb.) K. Schum. Borreria verticillata (L.) C.F.W. Mey. Cephalanthus glabratus (Spreng.) K.

Schum.

Coccocypselum lanceolatum (R. \& P.)

Pers.

Diodia alata Nees. \& Mart.

Diodia brasiliensis Spreng.

$x$

poaia-comprida

sarandi-branco

$\mathrm{x}$

Diodia saponariifolia (Cham. et Schlecht.) 


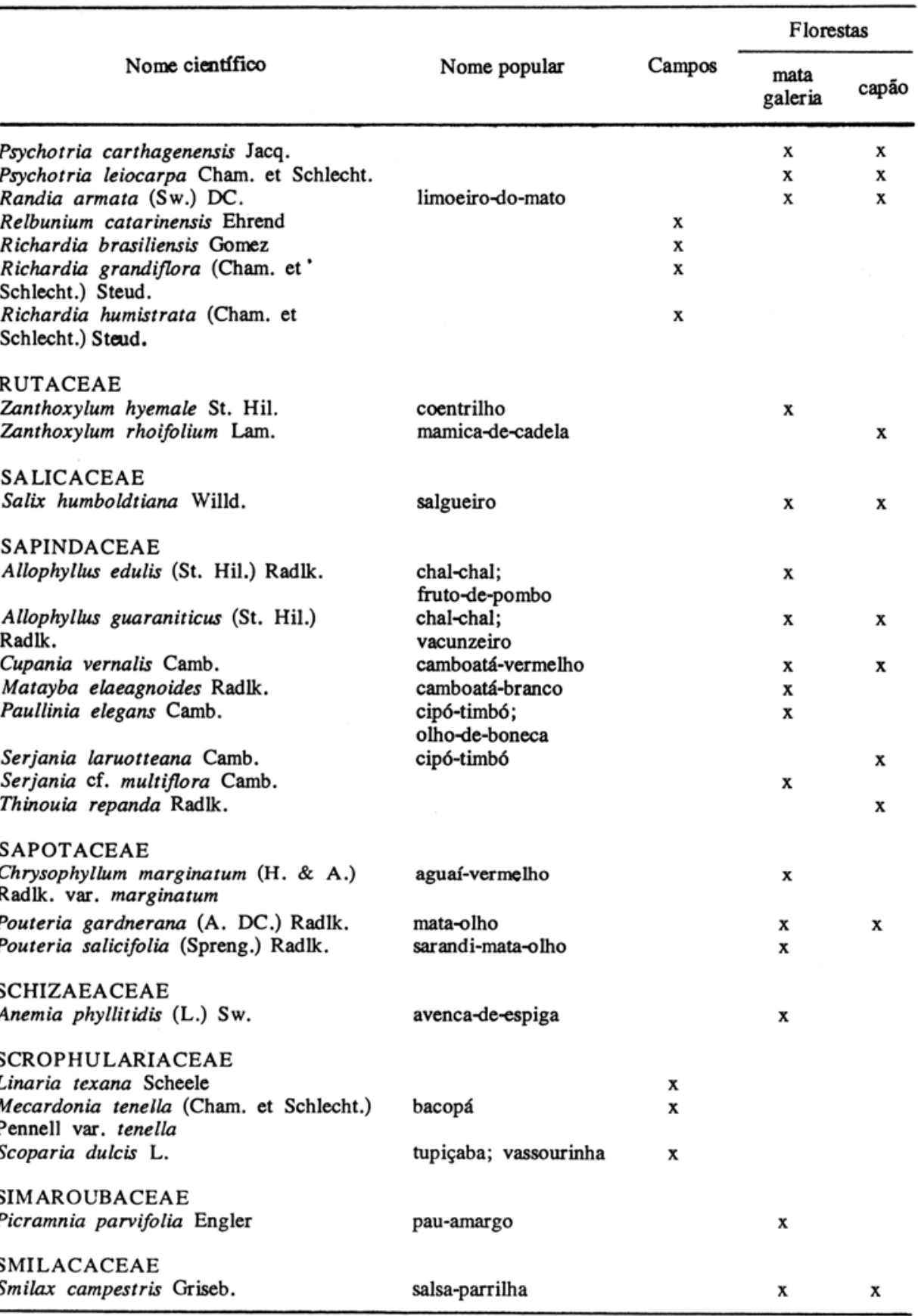


Florestas

Nome cientifico
Nome popular

\section{Campos}

pimenta-braba

X

Capsicum flexuosum Sendtn.

Petunia ovalifolia Miers

Petunia integrifola (Hook.)

Schinz \& Thell.

Solanum diflorum Vell.

Solanum inaequale Vell.

Solanum cf. inodorum Vell.

Solanum reflexum Schrank

Solanum sanctae-catharinae Dunal

Solanum sisymbriifolium Lam.

Solanum sp.

STERCULIACEAE

Byttneria urticifolia K. Schum.

SYMPLOCACEAE

Symplocos uniflora (Pohl) Benth.

\section{THYMELAEACEAE}

Daphnopsis racemosa Griseb.

\section{TILIACEAE}

Luehea divaricata Mart.

Triumfetta sp.

\section{TURNERACEAE}

Piriqueta selloi Urban

Turnera sp.

\section{ULMACEAE}

Celtis tala Gill. ex Planch.

UMBELLIFERAE

Apium leptophyllum (Pers.) F. Muell.

Centella asiatica (L.) Urban

Eryngium ciliatum Cham. et Schlecht.

Eryngium cf. eburneum Dcne.

Eryngium horridum Malme

Eryngium pandanifolium Cham. et

Schlecht.

Hydrocotyle leucocephala Cham. et

Schlecht.

\section{VERBENACEAE}

Glandularia peruviana (L.) Small

Stachytarpheta cayennensis (L.C.Rich.)

Vahl

$\begin{array}{ll}\text { peloteira; tomatinho } & \times \\ \text { capitão-do-campo; } & \times \\ \text { canema } & \times \\ & \times \\ \text { joá } & \times \\ \end{array}$

$x$

$x$

$x$

$\mathrm{x}$

$x$

$\mathrm{x}$

$\mathbf{x}$

$\mathrm{x}$

$\mathrm{x}$

raspa-canela

$\mathrm{x}$

sete-sangrias

$\mathrm{x}$

embira

$\mathrm{x}$

x

açoita-cavalo

$\mathrm{x}$

$\mathrm{x}$

$\mathrm{x}$

$\mathrm{x}$

$\mathbf{x}$

esporāo-de-galo

$\mathbf{x}$

$\mathrm{x}$

pé-de-cavalo

$\mathrm{x}$

gravatá-do-campo

caraguatá

caraguatá-do-

banhado

erva-capitāo-miúdo x gervão

X

x 


\begin{tabular}{|c|c|c|c|c|}
\hline \multirow[b]{2}{*}{ Nome científico } & \multirow[b]{2}{*}{ Nome popular } & \multirow[b]{2}{*}{ Campos } & \multicolumn{2}{|c|}{ Florestas } \\
\hline & & & $\begin{array}{l}\text { mata } \\
\text { galeria }\end{array}$ & capão \\
\hline $\begin{array}{l}\text { Verbena litoralis H.B.K. } \\
\text { Vitex megapotamica (Sprerig.) Moldenke }\end{array}$ & $\begin{array}{l}\text { erva-do-pai-Caetano } \\
\text { tarumã }\end{array}$ & $\mathrm{x}$ & $\mathbf{x}$ & \\
\hline $\begin{array}{l}\text { VIOLACEAE } \\
\text { Hybunthus sp. }\end{array}$ & & $\mathrm{x}$ & & \\
\hline $\begin{array}{l}\text { XYRIDACEAE } \\
\text { Xyris jupicai L.C. Rich. var. jupicai }\end{array}$ & & $\mathbf{x}$ & & \\
\hline
\end{tabular}

\section{Conclusões}

Pode-se observar que a cobertura vegetal da área foi sensivelmente alterada pela utilização do solo com culturas cíclicas ou permanentes, além do pastoreio sobre os campos, assim como pela extração de essências mais valiosas dos capões e matas galeria.

Face a pequena área ocupada, ainda, por florestas e a ocorrência de enchentes periódicas, é necessário que a vegetação florestal seja mantida, considerando-se a sua importância na contenção da erosão das margens dos rios e arroios.

Em termos florísticos, a vegetação florestal da área estudada faria parte da Floresta Estacional Decidual Aluvial (KLEIN 1983) que reveste as várzeas e terraços aluviais da Bacia do Jacuí e seus afluentes, sendo maior o contingente de espécies da Floresta Subtropical do Alto Uruguai, tendo-se constatado, também, a ocorrência de elementos característicos da Floresta Pluvial da Encosta A tlântica segundo KLEIN (1983), como Ficus organensis, Myrcia glabra, Nectandra rigida, Psychotria carthagenensis, Coussapoa schottii, Faramea marginata e Bactris lindmaniana. Também são elementos dessa Floresta, (WINKLER, 1980) os epífitos: Tillandsia geminiflora e $T$. stricta, enquanto que Bilbergia zebrina é representante da Floresta Pluvial Subtropical.

\section{Agradecimentos}

As autoras agradecem ao Dr. Roberto Miguel Klein, do Herbário Barbosa Rodrigues, pela consultoria, orientação e ensinamentos dados a campo, e ao Prof. Jorge Luiz Waechter, do Departamento de Botânica da Universidade Federal do Rio Grande do Sul pelas valiosas sugestões. 


\section{Referências Bibliográficas}

BRASfL, Ministério da Agricultura \& Rio Grande do Sul. Secretaria da Agricultura. 1970. Levantamento de reconhecimento dos solos do Estado do Rio Grande do Sul. Porto Alegre. Imapa. Escala 1:750.000.

FUNDAÇĀO ZOOBOTÂNICA DO RIO GRANDE DO SUL. Museu de Ciências Naturais. 1981. Estudo de auto-ecologia e bioindicadores de Poluiçâo visando a preservação das condiçóes ambientais na área do III Pólo Petroquímico; relatório técnico. Porto Alegre.

INCRA. 1972. Geomorfologia - Rio Grande do Sul. Rio de Janeiro. INCRA/IICA. Escala $1: 100.000$.

INCRA. 1973. Levantamento e avaliaçẩo de recursos naturais sócio-econômicos e institucionais do Rio Grande do Sul. Brasília. v.2.

KLEIN, R.M. 1983. Aspectos fitofisionômicos da Floresta Estacional na fralda da Serra Geral (RS). In: CONGRESSO NACIONAL DE BOTÂNICA, 34.', Porto Alegre, 1983. Anais..., v.1, p.73-110.

KOEPPEN, W. 1948. Climatologia: con un estudio de los climas de la tierra. México, Fondo de Cultura Economico. 478p.

LINDMAN, C.A.M. 1906. A vegetaçâo no Rio Grande do Sul. Porto Alegre. Liv. Universal. $356 \mathrm{p}$. , il.

MOTTA, F.S. 1951. Estudos do clima do Rio Grande do Sul segundo Sistema de W. Koeppen. Rev. bras. geogr.: 13(2): 107-16.

RAMBO, B. 1956. A fisionomia do Rio Grande do Sul. 2.ed. rev. Porto Alegre, Selbach. 456p. (Jesuítas no Sul do Brasil, 6).

SAINT-HILAIRE, A. de. 1939. Viagem ao Rio Grande do Sul (1820-1821). São Paulo. Ed. Nacional, 40p. (Brasiliana. Série 5).

WINKLER, S. 1980. Ursachen der Verbreitungsmuster einiger Bromeliaceae in Rio Grande do Sul (Südbrasilien). Flora, 170: 371-393. 